\title{
The Preparation of Texture Specimens of Aluminium
}

\author{
P. I. WELCH
}

Institute für Metallkunde und Metallphysik, Technische Universität, Clausthal, FRG.

This work was carried out while the author was in the Department of Metallurgy, University of Cambridge.

\section{INTRODUCTION}

Diffraction from the crystallographic lattice planes of a polycrystalline metal is a technique often used in the determination of crystallographic preferred orientation, or texture. Owing to its wide availability X-ray diffraction is a commonly used technique for this purpose. A characteristic of X-rays at normal operating wavelengths is their comparatively low penetration depth of a few microns in most metals. Thus usually a layer of a few grains at most is sampled. This necessitates the preparation of a flat, strain-free, surface for the measurement. Usually this is achieved by a combination of mechanical abrasion followed by mechanical polishing and etching, care being taken to avoid the fairly common orientation sensitive etches.

The availability of the Crystallite Orientation Distribution Function (CODF) (Roe, 1966; Bunge, 1969) has given a more precise description of textures than was possible with polefigures and has at the same time presented a sensitive tool for the investigation of the effect of measurement parameters on the textures measured.

\section{EXPERIMENTAL}

A commercial purity aluminium sample was rolled $70 \%$ on a 2 -High laboratory rolling mill with $15 \mathrm{~cm}$ diameter work rolls. Care was taken, by suitable lubrication and reversal during rolling, to ensure as homogeneous a texture distribution as possible. A composite sample was produced from 
this material using the technique described by Welch (1980). The same sample was prepared for measurement in three different conditions:

1) surface milled with normal milling cutter

2) subsequently ground on successive grades of corundum paper up to grade 600 grit

3) as 2 but subsequently polished using $6 \mu$ and $1 \mu$ diamond

after each treatment the speciment was chemically polished in a mixture of $5 \%$ Nitric acid, $70 \%$ Ortho-Phosphoric acid and $25 \%$ Sulphuric acid at $80^{\circ} \mathrm{C}$. The acids were used in the normal commercial concentrated form. Subsequent to each polishing the specimen was measured by $\mathrm{Cu}-\mathrm{K}_{\alpha} \mathrm{X}$-rays in reflection, the (111) (200) and (220) polefigures being measured. Because of the composite nature of the specimen, a complete quadrant of the polefigure could be measured in the reflection mode. The specimens were measured in the order given so that as far as possible the same sample volume was measured. Previous experience had confirmed that with the rolling procedure adopted successive samples from the same sheet were identical, as far as could be judged from the resulting CODFs.

\section{RESULTS}

The three sets of polefigures were analysed mathematically to generate the CODFs. These are shown in Figures 1-3. The dotted line corresponds to that intensity which would be expected for a random sample. All three show an F.C.C. rolling texture orientation tube running from an orientation $\{110\}\langle 112\rangle$ at $\phi=0^{\circ}, \psi=54.7^{\circ}, \theta=45^{\circ}$ to one at $\left\{\begin{array}{lll}4 & 11\end{array}\right\}\langle 11118\rangle$ at $\phi=45^{\circ}, \psi=0^{\circ}, \theta=32^{\circ}$. This is the generally accepted form of the cold rolling texture of aluminium of this purity (Welch, 1977). Figures 2 and 3 show an almost identical texture but the texture from the milled sample in Figure 1 shows a considerably lower severity. The maxima are in the same positions but the peaks are lower and much of the fine detail is lost. The general features of the texture are, however, the same and there are no significant texture components which are not common to both. The distribution of the components along the skeletal line of the orientation tube is also very similar but at a different level (Figure 4).

\section{DISCUSSION}

The experiment was conducted as part of a series to standardise procedures for CODF measurements. It was concluded that for aluminium the final 

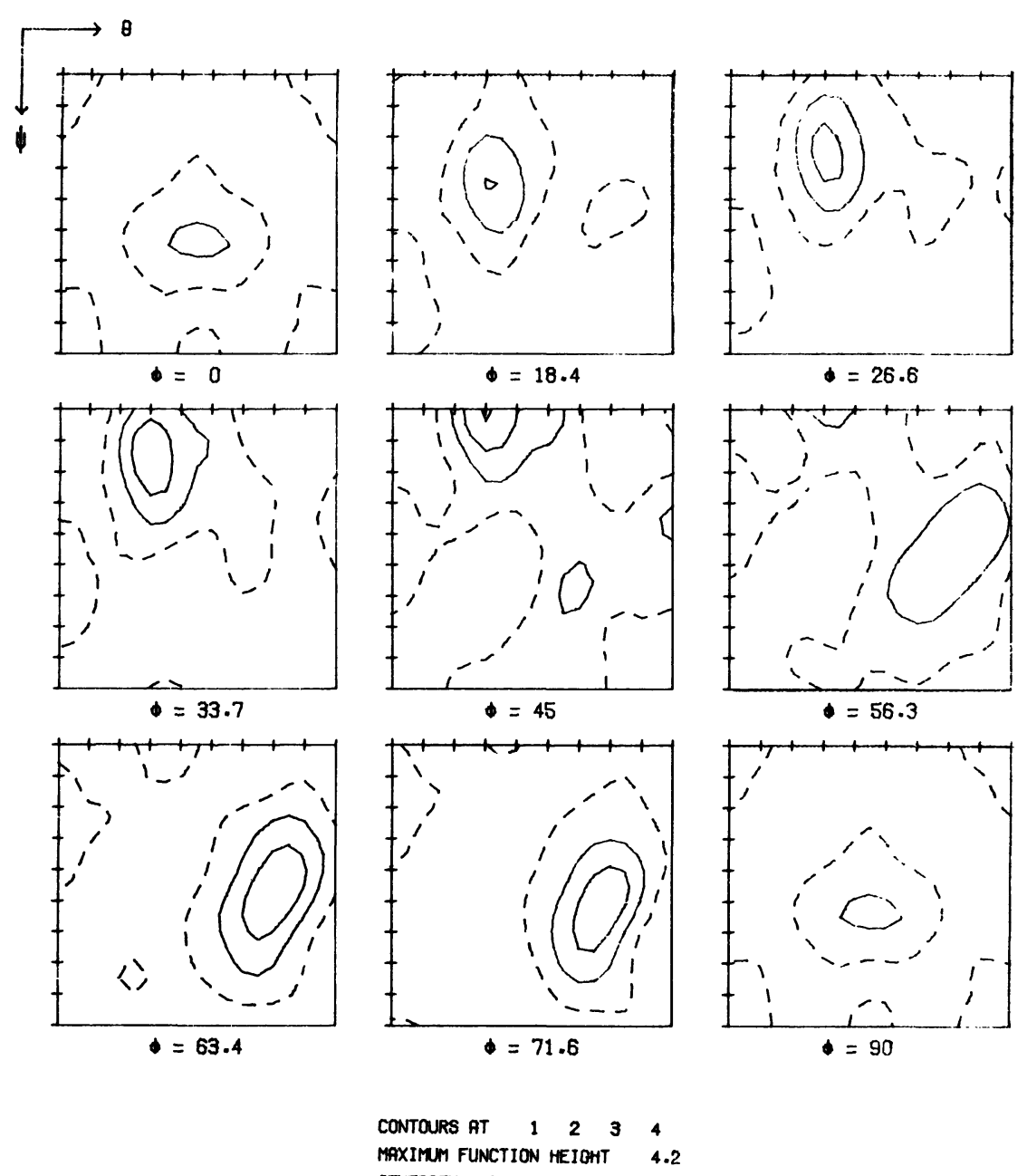

SEVERITY $\quad 0.71$

FIGURE 1 CODF for commercial purity aluminium sample rolled $70 \%$, surface milled prior to chemical polish and examination. 

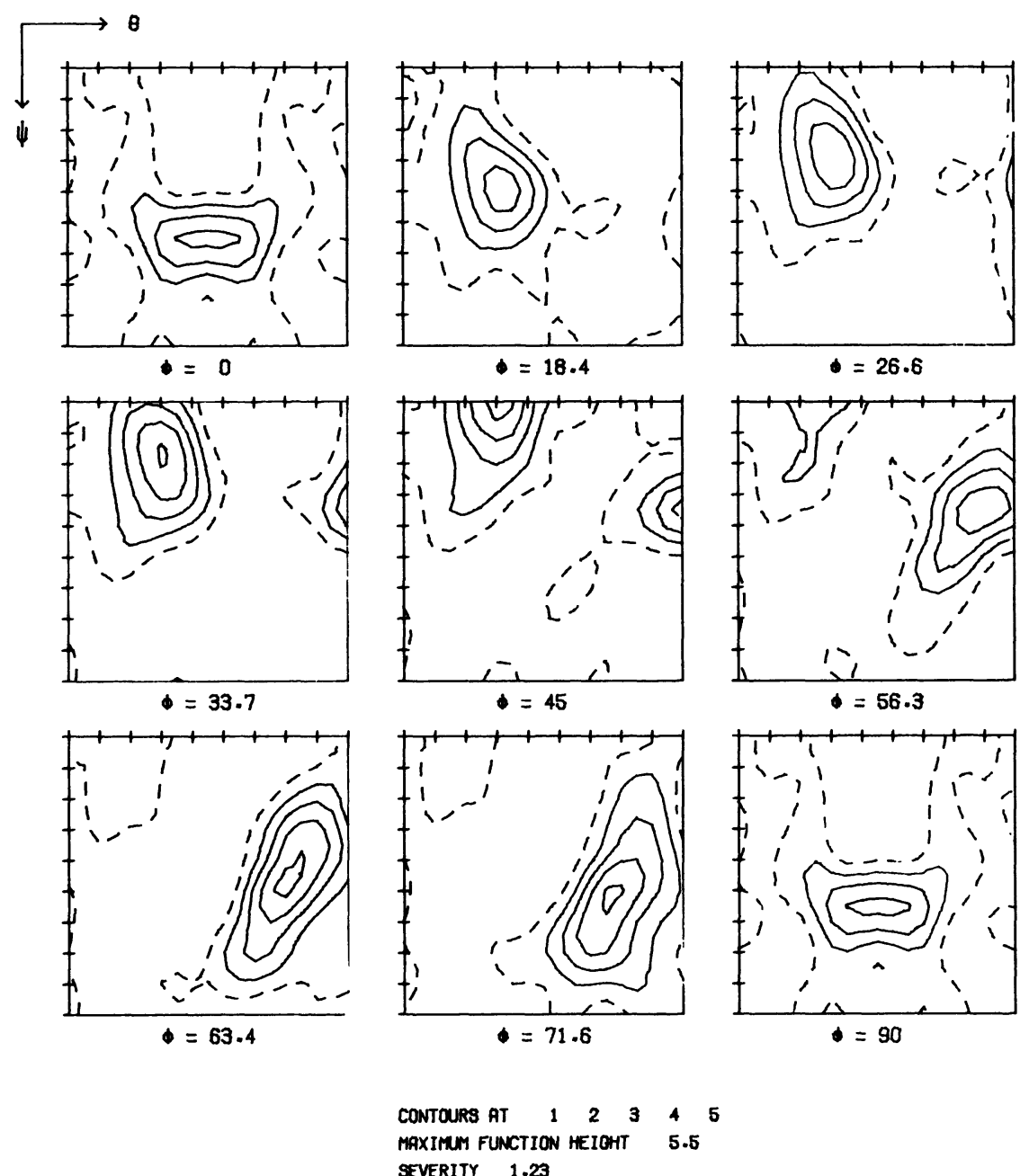

FIGURE 2 CODF for specimen as Figure 1 but ground on 600 grit prior to chemical polish. 

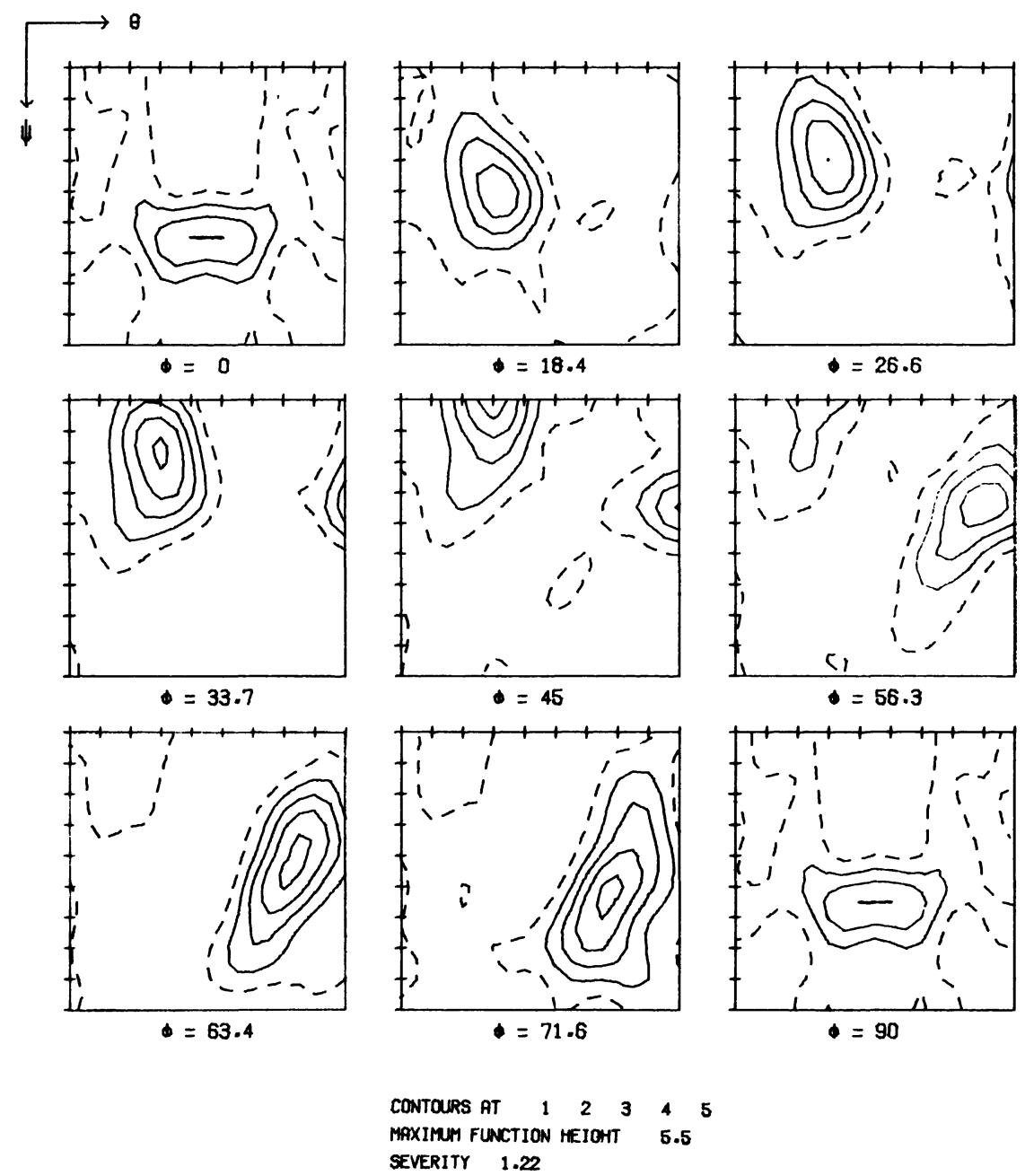

FIGURE 3 CODF for specimen as in Figure 2 polished on $6 \mu$ and $1 \mu$ diamond prior to chemical polish. 


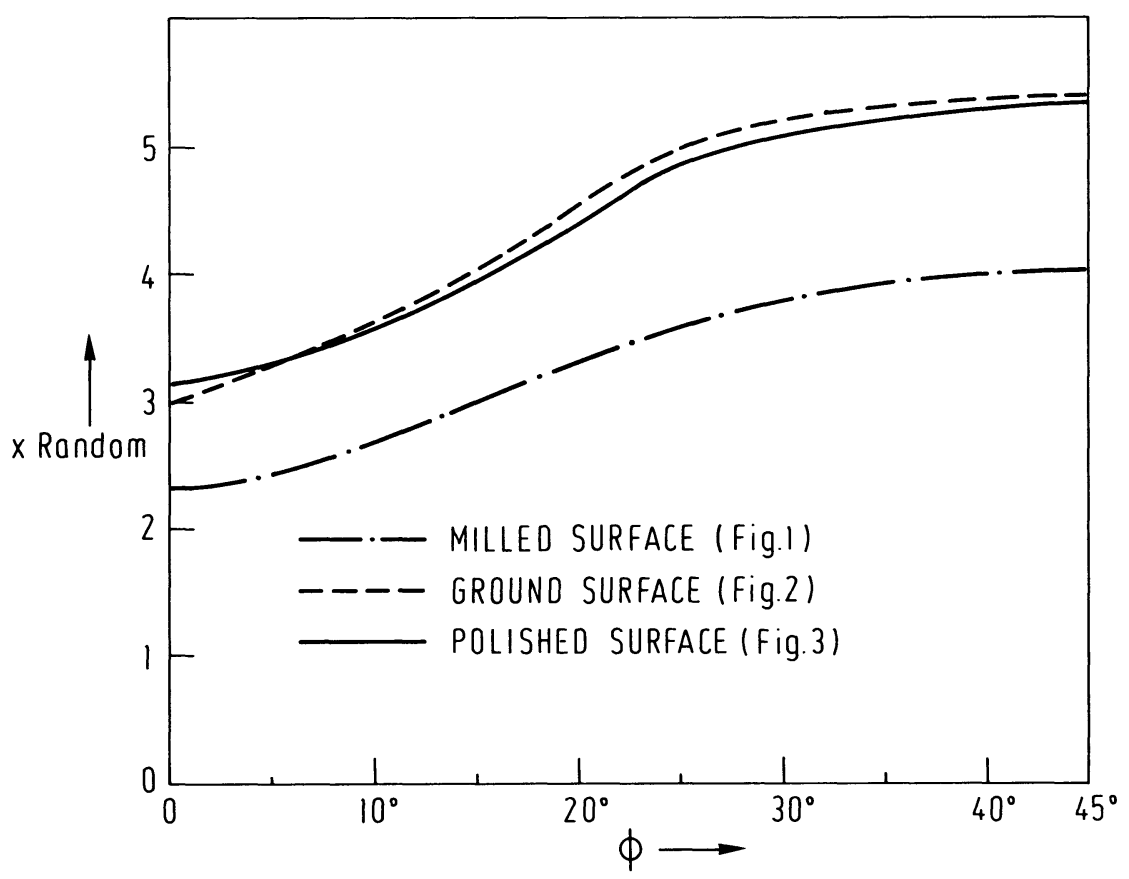

FIGURE 4 Orientation density along the skeletal line of the orientation tube for the three specimens.

mechanical polishing was unnecessary. The effect of doing without the mechanical abrasion as well, was characterised by a reduction in the sensitivity of the method but no significant change in the texture components measured was observed. This result has been confirmed by other workers (Bricknell, pers. comm.) where a surface prepared by a cut-off wheel had been measured directly, without a chemical polish, and measurements after fine grinding and chemical polish showed a somewhat sharpened texture but no relative change in the components present.

\section{CONCLUSIONS}

The effect of a rough surface in the X-ray measurement of aluminium textures was to reduce the severity of the measured texture but the distribution of components was unaffected.

Chemical polishing of aluminium specimens with the reagent used here renders the fine polishing stages, which occupy a completely disproportionate amount of specimen preparation time, unnecessary. 


\section{REFERENCES}

Bunge, H. J. Mathematische Methoden der Texturanalyse. Akedemie-Verlag, Berlin (1969).

Roe, R. J. J. Appl. Phys. 37, 2024-2069 (1966).

Welch, P. I. Texture, 4, 99 (1980).

Welch, P. I. Ph.D. Thesis, University of Cambridge (1977). 